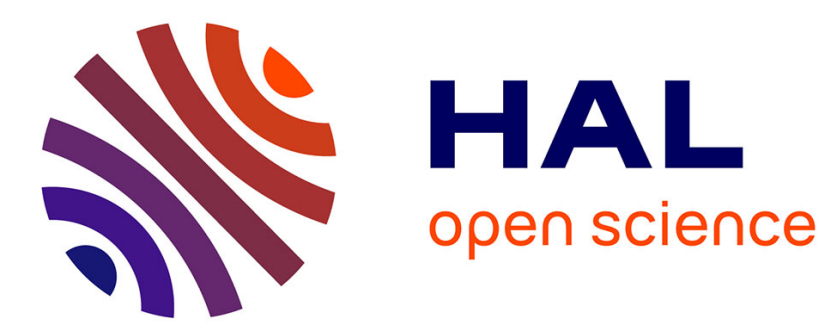

\title{
A Simple Altitude Capture Avoiding TCAS Resolution Advisories During Level-Off Situations
}

\author{
Thierry Miquel, Philippe Louyot, Bernard Hasquenoph
}

\section{To cite this version:}

Thierry Miquel, Philippe Louyot, Bernard Hasquenoph. A Simple Altitude Capture Avoiding TCAS Resolution Advisories During Level-Off Situations. AIAA GNC 2009, AIAA Guidance, Navigation and Control Conference, Aug 2009, Chicago, United States. pp xxxx, 10.2514/6.2009-5986 . hal00998701

\section{HAL Id: hal-00998701 \\ https://hal-enac.archives-ouvertes.fr/hal-00998701}

Submitted on 2 Jun 2014

HAL is a multi-disciplinary open access archive for the deposit and dissemination of scientific research documents, whether they are published or not. The documents may come from teaching and research institutions in France or abroad, or from public or private research centers.
L'archive ouverte pluridisciplinaire HAL, est destinée au dépôt et à la diffusion de documents scientifiques de niveau recherche, publiés ou non, émanant des établissements d'enseignement et de recherche français ou étrangers, des laboratoires publics ou privés. 


\title{
A Simple Altitude Capture Avoiding TCAS Resolution Advisories During Level-Off Situations
}

\author{
Thierry Miquel* \\ Direction des Services de la Navigation Aérienne, Toulouse, France \\ Philippe Louyot ${ }^{\dagger}$ \\ Direction des Services de la Navigation Aérienne, Toulouse, France \\ and \\ Bernard Hasquenoph \\ Egis avia, Toulouse, France
}

\begin{abstract}
TCAS resolution advisories generation process during level-off situation is overviewed and illustrated on a real situation captured through mode $S$ radar with RA downlink service. It underlines that one common type of resolution advisories is that which is issued when aircraft are expected to level-off $\mathbf{1 0 0 0}$ feet apart, and are converging at the same time. These resolution advisories, often subsequently classed as 'operationally unnecessary' from an air traffic control standpoint, can be perceived as disturbing by controllers and by pilots. This issue has been tackled in this paper by proposing a method to prevent RA triggering during level off situation. This method is based on the setting of the altitude at which the autopilot switches towards the altitude capture mode and on an intrinsic parameter of the autopilot altitude capture mode, namely its natural frequency. It is shown that applying the proposed method to the illustrative example prevents the issuance of resolution advisories when aircraft are expected to level-off $\mathbf{1 0 0 0}$ feet apart. The method which is presented is one of the simplest options, and allows altitude capture without triggering resolution advisories for vertical speeds up to $3000 \mathrm{feet} / \mathrm{min}$. Nevertheless, the time needed by the aircraft to capture the cleared flight level is increased. Consequently, future work will focus on improving the time needed to capture the cleared flight level under the constraint to keep positive the distance between the autopilot altitude capture dynamic and the boundary of the RA zone.
\end{abstract}

\section{Introduction}

$\mathrm{T}$ he Airborne Collision Avoidance System II (ACAS) is an essential component of the Air Traffic Management (ATM) system. An ACAS is an aircraft system based on secondary surveillance radar transponder signals which operates independently of ground-based equipment to provide advice to the pilot on potential conflicting aircraft that are equipped with SSR transponders ${ }^{1}$. It serves as a last resort safety net irrespective of any separation standards.

Through antennas, ACAS interrogates Mode C and Mode S transponders of all aircraft in the vicinity. Based upon the replies received, the system tracks the slant range, altitude (when available) and relative bearing of surrounding traffic. It is worth remembering that ACAS has no knowledge of both intruder and own aircraft clearance or intentions. Two types of ACAS are standardized, ACAS I and ACAS II. ACAS I only provides traffic advisories (TAs). Traffic advisories aim to help the pilot in the visual search for the intruder aircraft. ACAS II can issue both traffic advisories and resolution advisories (RAs). Resolution advisories are avoidance maneuvers in the vertical plane, displayed to the pilot, against one or several intruder aircraft. When the intruder aircraft is also fitted with an ACAS system, both ACAS co-ordinate their RAs through the Mode S data link, in order to select complementary resolution movements.

\footnotetext{
*PhD, Civil Aviation Design and Operation Engineer, Separation and Alerting Systems Unit, miquel@cena.fr

† Senior technical expert on ASAS and ACAS, Separation and Alerting Systems Unit, louyot@cena.fr

* Air Traffic Control expert, ACAS specialist, hasquenoph@cena.fr
} 
The ACAS II mandate applies world-wide (ICAO) for all civil fixed-wing turbine-engined aircraft with a maximum take-off mass exceeding $5,700 \mathrm{~kg}$, or a maximum approved passenger-seating configuration of more than 19. Carriage and operation of the ACAS II compliant equipment is mandatory worldwide since 1st January 2003.

TCAS II version 7.0 is the only equipment which complies fully with ACAS II standards and recommended practices, as published by ICAO. Therefore, TCAS II version 7.0 equipage is required to meet the ACAS II mandate. This version of TCAS II became available in 1999. When compared to the former version 6.04a, it improves TCAS compatibility with the air traffic control system. It also introduces compatibility with RVSM, and offers improvements in the vertical tracking of intruders, and a filtering of threats based on a forecast horizontal miss distance.

Resolution advisories are generated when ACAS II computes a risk of collision with another aircraft within the next 35 seconds (or less, depending on altitude and whether the aircraft is flying level or not) ${ }^{2}$. RA is issued when aircraft are horizontally converging and are expected to be at the same altitude at the same time. One common type of RA is issued even if aircraft are cleared to level-off 1000 feet apart. These types of RAs represents $30 \%$ of issued RAs; they are often classified as 'operationally unnecessary' from an ATC standpoint and can be perceived as disturbing by controllers and by pilots. In addition, they can induce a vertical deviation of the level aircraft (through coordinated RA).

The following figure presents a real encounter captured in France through mode S radar with RA-downlink service. The first graph presents the horizontal geometry of the encounter, and the second graph the flight level of both aircraft against time. Times at which RA downlink messages are received by the mode S radar are represented by dots. Finally, the last graph presents the horizontal range between aircraft as a function of time. It can be seen that the intruder aircraft with a track angle of 338 degrees is level at $F L 360$ whereas own aircraft is flying from the South West with a track of 25 degrees. There is a first level off at $F L 340$, and then a second level off is planned at $F L$ 350. Due to a high vertical speed, an RA is issued on board the ascending aircraft before leveling off at $F L 350$ because TCAS anticipates a collision risk within a remaining time less than 35 seconds. This occurs despite the provision of 1000 feet for vertical separation planned by air traffic control. It is worth noticing that in this particular example, the intruder aircraft does not experience any resolution advisory owing to a lower alarm threshold associated with level flight.
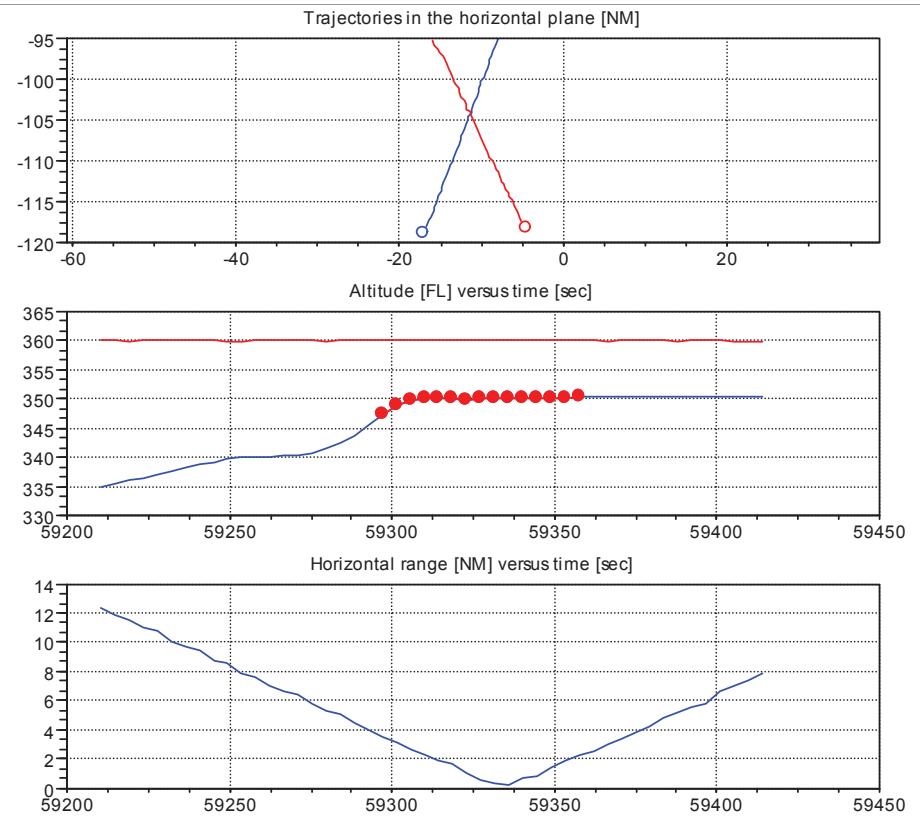

Fig. 1: Example of actual level off situation during which a resolution advisory is generated

The purpose of this paper is to design a simple autopilot altitude capture mode can be set to avoid resolution advisories during such level-off situations.

The paper is organized as follows: in the next section, the resolution advisories generation process during leveloff situation is overviewed and illustrated on a real situation captured through mode $\mathrm{S}$ radar with RA downlink service. The design of a simple autopilot altitude capture is addressed in the third section. This starts by the presentation of a simple altitude capture model. The simple altitude capture model is based on second order linear 
system theory. The identification process which enables to set the parameters of this model in order to reproduce the observed altitude capture is then described. The observed RA is reproduced thanks to a simulation process including aircraft and TCAS modeling. The setting of the simple autopilot altitude capture mode is addressed in the fourth section. Within that section, a method to prevent RA triggering during level off situation is presented. This method is based on the setting of the altitude at which the autopilot switches towards the altitude capture mode and the natural frequency $w_{n}$ of the altitude capture mode. This is done under the hypothesis that the product $m \cdot w_{n}$ remains constant so as not to modify the real part of the poles of the autopilot mode. This method is illustrated through the real radar encounter previously presented. Finally, the conclusion underlines that further work is required in order to extend the results to the cases where the intruder aircraft is not level.

\section{Overview of resolution advisories generation process during level-off situation}

The purpose of this section is to overview the resolution advisories generation process during level-off situation. It is illustrated by a real situation captured through mode $\mathrm{S}$ radar with RA downlink service. It is assumed in the following that own aircraft will level off after a climb (or a descent) and that the intruder aircraft is level.

TCAS tracks range of intruders and determines whether collision can occur within the given time threshold (range test). If the intruder passes the range test, TCAS determines whether the intruder is currently, or projected to be, close in altitude (altitude test). Assuming that the range test is passed, a resolution advisory is generated when the time to co-altitude test is less than a specific threshold (same threshold than for the range test). This threshold depends on altitude and whether the aircraft is flying level or not. The value of the threshold is 35 seconds for flight level higher than FL200, but is lower at lower altitudes.

Time to co-altitude test is determined by the values of own and intruder aircraft's current vertical rates. Under the assumption that the intruder aircraft is level, the computed time to co-altitude $\tau_{V}$ has the following expression, where $h$ and $V_{z}$ designate the altitude and vertical speed of own aircraft, and $h_{i c}$ the constant altitude of the intruder aircraft:

$$
\tau_{V}=-\left(\frac{h-h_{i c}}{V_{z}}\right)
$$

In the following, this relationship will be labeled 'Boundary of the RA zone' when the computed time to coaltitude $\tau_{V}$ is equal to the threshold $S$ at which an RA is triggered.

The dynamic of the altitude capture mode of the autopilot is now considered. Let $h_{c}$ be the selected altitude by own aircraft. When the dynamic of the altitude capture mode is superimposed on the domain $\left(V_{z} ; h\right)$ with the boundary of the RA zone, the following figure is obtained. It has been assumed that the selected altitude $h_{c}$ is higher than the altitude at which the capture starts. It is worth noticing that the slope of the straight line corresponds to the threshold at which an RA is triggered.

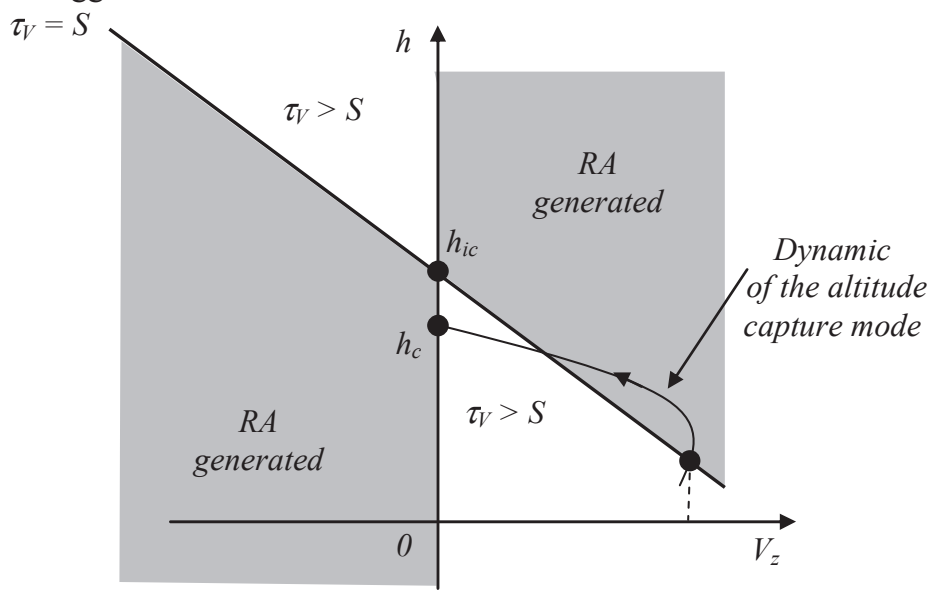

Fig. 2: Dynamic of the altitude capture mode superimposed on the domain where the computed time to coaltitude triggers an alarm

From the previous figure, it is clear that if the dynamic of the altitude capture mode enters the grey area which delimits the area where an alarm is triggered, then an RA is generated. In order to illustrate those relationships, the 
following figure presents the plot $V_{z} \rightarrow h\left(V_{z}\right)$ for the captured encounter presented during the introduction as well as the boundary of the RA zone, where threshold $S$ is set to 35 seconds and intruder flight level $h_{i c}$ is set to FL 360:

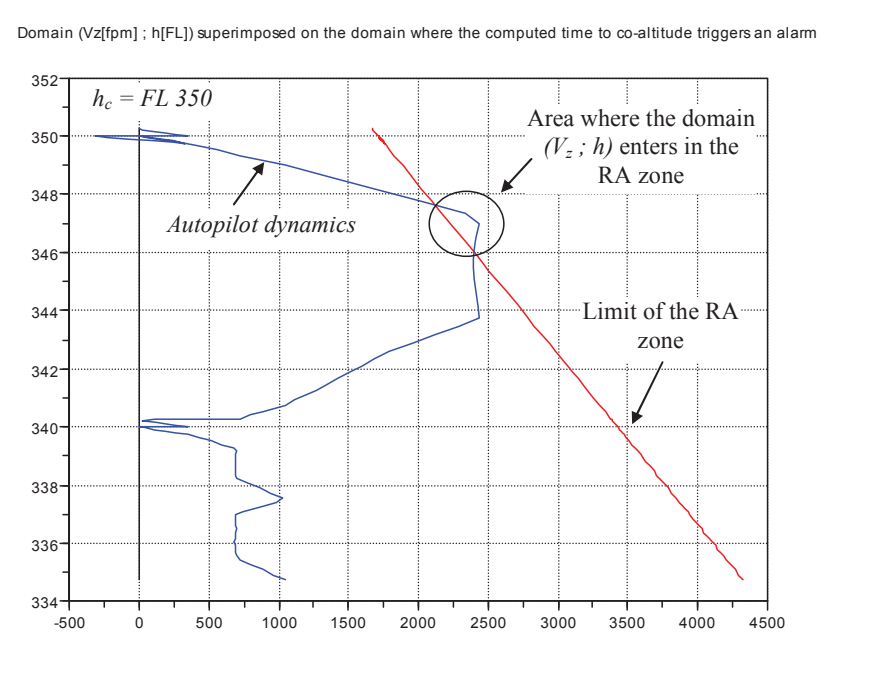

Fig. 3: Domain $\left(V_{z} ; h\right)$ for the captured trajectory and boundary of the RA zone

On this figure it is clearly seen that the aircraft achieves a vertical speed of about $2400 \mathrm{fpm}$ and then enters the RA zone before moving towards the cleared altitude, which is FL 350. Indeed, when the aircraft crosses $F L 346$, TCAS estimates the co-altitude time with the intruder to be 35 seconds $(F L 360-2400 \mathrm{fpm} \times 35 \mathrm{sec}=F L 346)$ and then triggers an RA. Consequently, a way to avoid RAs triggering is to modify the dynamic of the altitude capture mode so that it always remains outside the area where an RA is generated. This general idea will be investigated in the following paragraph.

\section{Simple altitude capture autopilot design}

\section{A. Aircraft model}

As far as the aircraft dynamic is considered, the following assumptions are made:

- Flat, non-rotating earth, standard atmosphere and constant gravity;

- The aircraft is regarded as a point mass: the dynamics of the movements around its centre of mass are ignored;

- The aircraft is flying a straight line at constant equivalent airspeed and is controlled through load factor, that is by the ratio between lift and weight.

Taking into account the above assumptions, the longitudinal point mass dynamic equations of motion referring to the Earth fixed reference frame are the following ${ }^{3}$, where $x$ stand for the horizontal coordinates and $h$ for the vertical coordinates of the aircraft, $V_{a}$ for true airspeed, $V_{e}$ for equivalent airspeed, $g$ for gravitational acceleration, $\gamma$ for flight path angle, $n_{z}$ for load factor, and $\rho(0)$ and $\rho(h)$ for the air density respectively at sea level and at altitude $h$.

$$
\begin{aligned}
& \left\{\begin{array}{l}
\dot{x}=V_{a} \cdot \cos (\gamma) \\
\dot{h}=V_{a} \cdot \sin (\gamma) \\
\dot{\gamma}=\frac{g}{V_{a}} \cdot\left(n_{z}-\cos (\gamma)\right)
\end{array}\right. \\
& \left\{\begin{array}{l}
V_{a}=V_{e} \cdot \sqrt{\frac{\rho(0)}{\rho(h)}} \\
\dot{V}_{e}=0
\end{array}\right.
\end{aligned}
$$




\section{B. Design of the altitude capture mode}

The purpose of the altitude capture mode is to constrain the actual aircraft altitude $h$ to move towards the commanded altitude $h_{c}$. First, assuming a constant true airspeed $V_{a}$ and a flight path angle small enough such that $\sin (\gamma) \approx \gamma$, the second time derivative of the altitude $h$ with respect to the time is obtained from the last two equations of (2) and leads to an expression where control $n_{z}$ appears:

$$
\ddot{h} \approx V_{a} \cdot \dot{\gamma}=g \cdot\left(n_{z}-\cos (\gamma)\right)
$$

Feedback linearization is then used: the altitude capture mode constrains the dynamic of the actual altitude $h$ to move towards the commanded altitude $h_{c}$ by following a second order stable differential equation with undamped natural frequency $w_{n}$ and damping ratio $m$ :

$$
\ddot{h}=-2 \cdot m \cdot w_{n} \cdot \dot{h}-w_{n}^{2} \cdot\left(h-h_{c}\right)
$$

Combining (4) and (5) and taking into account the approximation $\dot{h} \approx V_{a} \cdot \gamma$ leads to the expression of the control $n_{z}$

$$
n_{z}=\cos (\gamma)-\frac{2 \cdot m \cdot w_{n} \cdot V_{a} \cdot \gamma+w_{n}^{2} \cdot\left(h-h_{c}\right)}{g}
$$

\section{Parameter identification}

The estimation of the slope of the altitude capture mode (see on Fig. 3 above the arrow labeled 'autopilot dynamic') for the radar captured encounter is here considered. It is assumed that the altitude capture mode described in the previous section correctly described the aircraft behavior during the altitude capture. First, it is worth noticing that the dynamic (5) of the autopilot altitude capture mode can be rewritten as follows:

$$
\frac{1}{w_{n}^{2}} \cdot \ddot{h}+\frac{2 \cdot m}{w_{n}} \cdot \dot{h}+\left(h-h_{c}\right)=0
$$

In the following, it is assumed that the altitude capture starts around a point where the second derivative of the altitude $h$ is close to zero. This is assumed to be around the switching altitude where the altitude capture starts. This corresponds to the vertical segment in the domain $\left(V_{z} ; h\right)$ represented in Fig. 3 . Then, the dynamic of the altitude capture mode is approximated by the following first order dynamic:

$$
\frac{2 \cdot m}{w_{n}} \cdot \dot{h}+\left(h-h_{c}\right) \approx 0
$$

Let $T_{s}$ be the time at which RA appears and let the time interval $\left[T_{s}, T_{s}+5\right.$ seconds] be the identification window for the ratio $2 \cdot m / w_{n}$. The 5 seconds duration of the identification window may appear quite small, but is justified by the fact that this corresponds to the standard aircrew reaction time for initial $\mathrm{RA}^{5}$. A longer duration of the identification window may capture the dynamic of the manual control law rather than the dynamic of the altitude capture mode. In order to identify the ratio $2 \cdot m / w_{n}$ defined as parameter $p$, let $\varepsilon(p)$ be the following quadratic error:

$$
p=\frac{2 \cdot m}{w_{n}} \Rightarrow \varepsilon(p)=\frac{1}{2} \cdot \sum_{k}\left(p \cdot \dot{h}\left(t_{k}\right)+\left(h\left(t_{k}\right)-h_{c}\right)\right)^{2}
$$

The identification of parameter $p$ is done by minimizing the quadratic error $\varepsilon(p)$ with respect to $p$ :

$$
\frac{\partial \varepsilon(p)}{\partial p}=\sum_{k}\left(p \cdot \dot{h}\left(t_{k}\right)+\left(h\left(t_{k}\right)-h_{c}\right)\right) \cdot \dot{h}\left(t_{k}\right) \hat{=} 0
$$

Consequently, parameter $p$ is approximated as follows:

$$
p=-\frac{\sum_{k}\left(h\left(t_{k}\right)-h_{c}\right) \cdot \dot{h}\left(t_{k}\right)}{\sum_{k} \dot{h}^{2}\left(t_{k}\right)}
$$

When this identification process is applied to the radar captured encounter presented in the introduction, parameter $p$ is evaluated to be approximately 9 seconds. Damping ratio $m$ has been set to 0.8 in order to avoid oscillations during the altitude capture. As parameter $p$ has been evaluated to be 9 seconds, and as $p$ equals $2 \cdot m / w_{n}$, this implies that the natural frequency $w_{n}$ is evaluated to be $0.178 \mathrm{rad} / \mathrm{sec}$. Simulating the altitude capture thanks to aircraft model (2) and control law (6) from $T_{s}$ to the final time of the trajectory leads to the following $\left(V_{z} ; h\right)$ plot: 


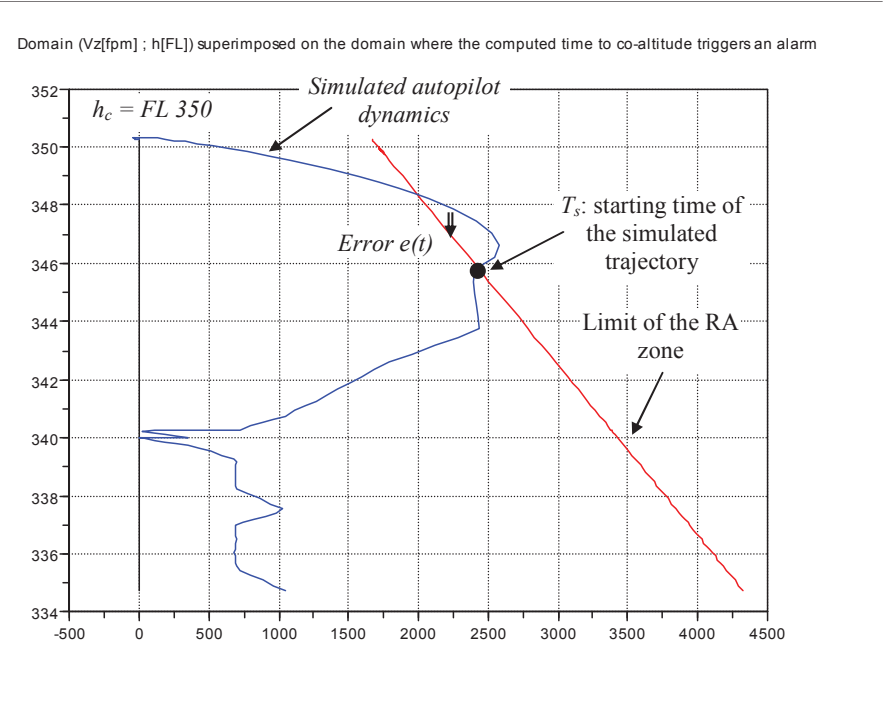

Fig. 4: Domain $\left(V_{z} ; h\right)$ for the simulated trajectory and boundary of the RA zone

From the previous figure, it is clear that the RA is reproduced through the simulation process and that the key factors which enable to avoid issuing an RA in such a situation are the altitude at which the autopilot switches towards the altitude capture mode as well as the parameters of the altitude capture mode itself, namely the damping ratio $m$ and the natural frequency $w_{n}$.

\section{Tuning the autopilot altitude capture mode to avoid RA during level off}

\section{A. Computation of the tangent to the boundary of the RA zone}

The second order filter (5) modeling the dynamic of the altitude capture mode can be written under its state form as follows:

$$
\left\{\begin{array}{l}
\underline{x} \hat{=}\left[\begin{array}{c}
\left(h-h_{c}\right) \\
\dot{h}
\end{array}\right] \\
A \hat{=}\left[\begin{array}{cc}
0 & -1 \\
w_{n}^{2} & 2 \cdot m \cdot w_{n}
\end{array}\right]
\end{array} \Rightarrow \underline{\dot{x}}=-A \cdot \underline{x}\right.
$$

Let $e(t)$ be the difference between the altitude limit of the RA zone (see (1)) and the altitude of the aircraft defined by (5), as represented in Fig. 4:

$$
e(t)=h_{i c}-S \cdot \dot{h}(t)-h(t)
$$

The error $e(t)$ can be expressed as a function of the state vector $\underline{x}$ as follows:

$$
e(t)=h_{i c}-h_{c}-S \cdot \dot{h}(t)-\left(h(t)-h_{c}\right)=h_{i c}-h_{c}-\left[\begin{array}{ll}
1 & S
\end{array}\right] \cdot\left[\begin{array}{c}
h(t)-h_{c} \\
\dot{h}(t)
\end{array}\right]=h_{i c}-h_{c}-\left[\begin{array}{ll}
1 & S
\end{array}\right] \cdot \underline{x}(t)
$$

When (12) is used, the time derivative of the error $e(t)$ has the following expression:

$$
\dot{e}(t)=-\left[\begin{array}{ll}
1 & S
\end{array}\right] \cdot \underline{\dot{x}}(t)=\left[\begin{array}{ll}
1 & S
\end{array}\right] \cdot A \cdot \underline{x}(t)=\left[S \cdot w_{n}^{2} \quad\left(2 \cdot m \cdot S \cdot w_{n}-1\right)\right] \cdot \underline{x}(t)
$$

As a consequence, the value of the state vector $x_{t}$ tangent to the RA zone satisfies the following set of equations:

$$
\left\{\begin{array} { l } 
{ e = 0 } \\
{ \dot { e } = 0 }
\end{array} \Leftrightarrow \left\{\begin{array}{l}
h_{i c}-h_{c}-\left[\begin{array}{ll}
1 & S
\end{array}\right] \cdot \underline{x}_{t}=0 \\
{\left[\begin{array}{ll}
S \cdot w_{n}^{2} & \left(2 \cdot m \cdot S \cdot w_{n}-1\right)
\end{array}\right] \cdot \underline{x}_{t}=0}
\end{array} \Leftrightarrow\left[\begin{array}{cc}
1 & S \\
S \cdot w_{n}^{2} & \left(2 \cdot m \cdot S \cdot w_{n}-1\right)
\end{array}\right] \cdot \underline{x}_{t}=\left[\begin{array}{c}
h_{i c}-h_{c} \\
0
\end{array}\right]\right.\right.
$$

Denoting by $V_{z t}$ the vertical speed at the tangent point and by $h_{t}$ the altitude at that point, we finally get:

$$
\begin{aligned}
& \underline{x}_{t}=\frac{1}{\left(S \cdot w_{n}\right)^{2}-2 \cdot m \cdot S \cdot w_{n}+1} \cdot\left[\begin{array}{cc}
1-2 \cdot m \cdot S \cdot w_{n} & S \\
S \cdot w_{n}^{2} & -1
\end{array}\right] \cdot\left[\begin{array}{c}
h_{i c}-h_{c} \\
0
\end{array}\right] \\
& \Leftrightarrow \underline{x}_{t} \hat{=}\left[\begin{array}{c}
h_{t}-h_{c} \\
V_{z t}
\end{array}\right]=\frac{h_{i c}-h_{c}}{\left(S \cdot w_{n}\right)^{2}-2 \cdot m \cdot S \cdot w_{n}+1} \cdot\left[\begin{array}{c}
1-2 \cdot m \cdot S \cdot w_{n} \\
S \cdot w_{n}^{2}
\end{array}\right]
\end{aligned}
$$


From the previous equation, the relationship between the altitude $h_{t}$ and the vertical speed $V_{z t}$ at the point where the altitude capture dynamic is tangent to the boundary of the RA zone is the following:

$$
\frac{h_{t}-h_{c}}{V_{z t}}=\frac{1-2 \cdot m \cdot S \cdot w_{n}}{S \cdot w_{n}^{2}}
$$

In addition, as far as $\underline{x}_{t}$ belongs to the trajectory defined by the differential equation (12), the set of initial conditions $\underline{x}_{0 t}$ for which the altitude capture trajectory tangents the boundary of the RA zone is given by:

$$
\underline{x}_{0 t}=\exp (-A \cdot t) \cdot \underline{x}_{t} \Leftrightarrow \underline{x}_{0 t}=\frac{h_{i c}-h_{c}}{\left(S \cdot w_{n}\right)^{2}-2 \cdot m \cdot S \cdot w_{n}+1} \cdot \exp (-A \cdot t) \cdot\left[\begin{array}{c}
1-2 \cdot m \cdot S \cdot w_{n} \\
S \cdot w_{n}^{2}
\end{array}\right]
$$

The expression of the matrix $\exp (-A \cdot t)$ can be found by using the Laplace transform ${ }^{6}$. In the following expression, $s$ represents the Laplace variable, and $I$ the identity matrix:

Where

$$
\exp (-A \cdot t)=L^{-1}\left[(s \cdot I+A)^{-1}\right]=\left[\begin{array}{ll}
a_{11}(t) & a_{12}(t) \\
a_{21}(t) & a_{22}(t)
\end{array}\right]
$$

$$
\left\{\begin{array}{l}
a_{11}(t)=-\frac{\sin \left(w_{n} \cdot t \cdot \sqrt{1-m^{2}}-\phi\right)}{\sin (\phi)} \cdot \exp \left(-m \cdot w_{n} \cdot t\right) \\
a_{12}(t)=\frac{\sin \left(w_{n} \cdot t \cdot \sqrt{1-m^{2}}\right)}{w_{n} \cdot \sqrt{1-m^{2}}} \cdot \exp \left(-m \cdot w_{n} \cdot t\right) \\
a_{12}(t)=-w_{n}^{2} \cdot a_{12}(t)=-w_{n} \cdot \frac{\sin \left(w_{n} \cdot t \cdot \sqrt{1-m^{2}}\right)}{\sqrt{1-m^{2}}} \cdot \exp \left(-m \cdot w_{n} \cdot t\right) \\
a_{22}(t)=\frac{\sin \left(w_{n} \cdot t \cdot \sqrt{1-m^{2}}+\phi\right)}{\sin (\phi)} \cdot \exp \left(-m \cdot w_{n} \cdot t\right) \\
\sin (\phi)=\sqrt{1-m^{2}} \\
\cos (\phi)=-m
\end{array}\right.
$$

The use of formulas (19) - (21) in association with the values of the natural frequency $w_{n}$ and damping ratio $m$ of the altitude capture mode derived in the previous section leads to the following figure. The altitude capture trajectory tangents to the boundary of the RA zone at time $t=0$ :

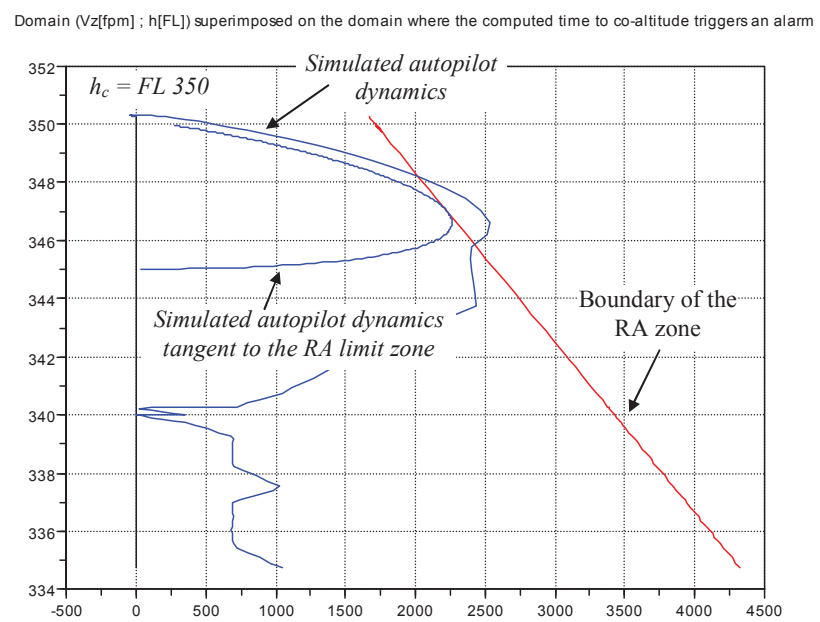

Fig. 5: Simulated autopilot dynamic which tangents the boundary of the RA zone

\section{B. Tuning the parameters of the altitude capture mode}

It is clear from Fig. 5 that the actual values of the natural frequency $w_{n}$ and damping ratio $m$ of the altitude capture mode do not allow to find some initial conditions (i.e. altitude / vertical speed at which the altitude capture 
mode switches on) which avoid an RA. Indeed, no intersection point exists between the autopilot dynamic which tangents the boundary of the RA zone and the trajectory $\left(V_{z} ; h\right)$ which precedes the starting of the altitude capture (i.e. the points $\left(V_{z} ; h\right)$ which are situated before starting time $\left.T_{s}\right)$.

As a consequence, the actual values of the natural frequency $w_{n}$ and damping ratio $m$ shall be modified in order to satisfy the following constraints: firstly, $w_{n}$ and $m$ shall be chosen such that the second order control system is always outside of the RA zone; and secondly an intersection point shall exist between the autopilot altitude capture dynamic and the trajectory $\left(V_{z} ; h\right)$ which precedes the engagement of the altitude capture mode (and which is which is situated before starting time $T_{s}$ ).

In order to satisfy both constraints, one of the simplest options consists in considering the maximum value of the vertical speed achievable by the aircraft, denoted $V z_{\max }$. The second time derivative of the altitude is then constrained to be zero at $V z_{\max }$. According to (5), this leads to the lowest value of the altitude capture (or the highest value if the aircraft is descending before the altitude capture), which is denoted $h\left(V z_{\max }\right)$ :

$$
0=-2 \cdot m \cdot w_{n} \cdot V z_{\max }-w_{n}^{2} \cdot\left(h\left(V z_{\max }\right)-h_{c}\right) \Rightarrow h\left(V z_{\max }\right)=h_{c}-\frac{2 \cdot m}{w_{n}} \cdot V z_{\max }
$$

Then, the point $\left(h\left(V z_{\max }\right), V z_{\max }\right)$ is constrained to belong to the trajectory defined by (19). That means that a time $t_{e}$ shall exist such that the following relationship holds:

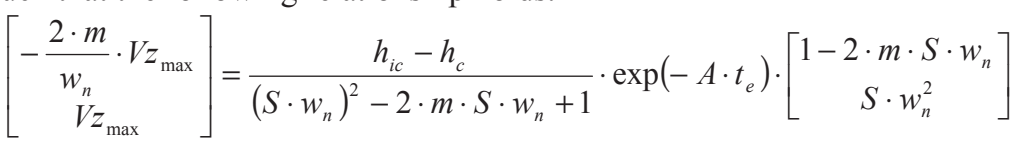

The following figure illustrates the second order filter dynamic which satisfies (23):

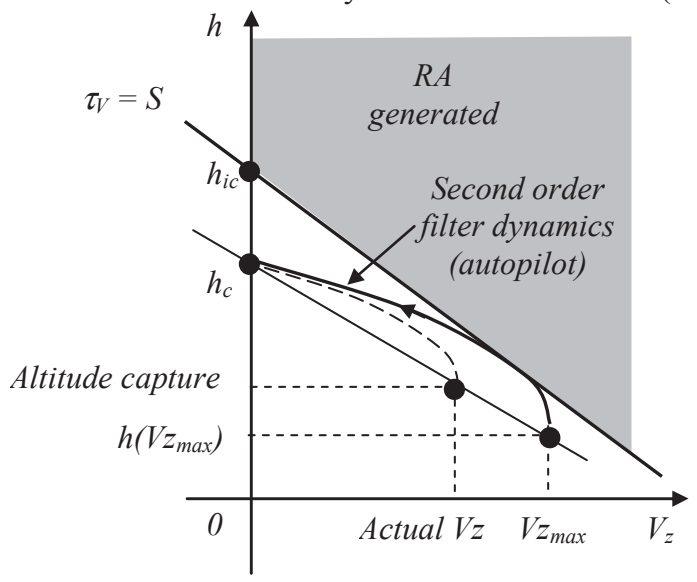

Fig. 6: Second order filter dynamic which tangents the boundary of the RA zone

As far as the actual vertical speed of the aircraft during the climb (or descent) is lower (respectively greater) than the maximum vertical speed $V z_{\max }$, the uniqueness ${ }^{4}$ of the solution of the differential equation (12) ensures that actual dynamic of the altitude capture mode will never enter inside the area where an RA is generated.

In addition, equation (22) indicates that the altitude capture mode is switched on as far as the following inequality holds, where $h_{c}$ is the commanded altitude and $V z$ the actual vertical speed during climb (or descent):

$$
\left|h-h_{c}\right|<\frac{2 \cdot m}{w_{n}} \cdot|V z|
$$

\section{Case study}

For the case study, it will be assumed that the product $m \cdot w_{n}$ remains constant in order not to modify the real part of the poles of the previously identified autopilot mode. Then, the maximum value of the vertical speed achievable by the aircraft, that is $V z_{\max }$., is set to $3000 \mathrm{feet} / \mathrm{min}$. Setting $h_{i c}-h_{c}$ to 1000 feet (that is the vertical separation standard between aircraft provided by Air Traffic Control), the threshold $S$ to 35 seconds (that is the upper bound of the threshold used by TCAS) and keeping the product $m \cdot w_{n}$ to $0.8 \cdot 0.178=0.142 \mathrm{rad} / \mathrm{sec}$, the natural frequency $w_{n}$ which matches equation (23) is found to be $0.134 \mathrm{rad} / \mathrm{sec}$ (which is about $25 \%$ lower than the natural frequency which has been estimated in the previous section, that is $0.178 \mathrm{rad} / \mathrm{sec}$ ), whereas the damping ratio $\mathrm{m}$ moves from 0.8 to 1.06 (in order to keep constant the product $m \cdot w_{n}$ ). 
The following figure presents the domain $\left(V_{z} ; h\right)$. The simulation of the control law (6) coupled with the aircraft model (2) starts at FL 344 in accordance with (24). This is 600 feet under the cleared flight level.

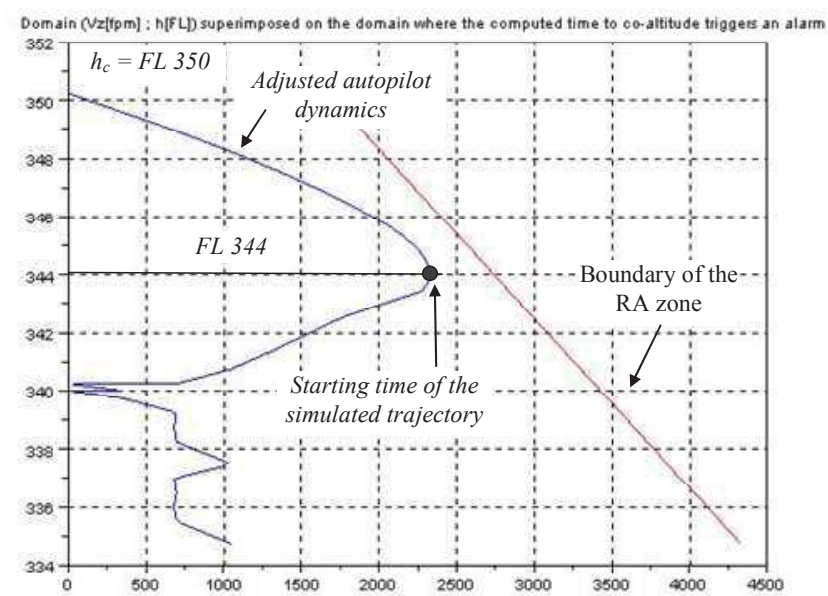

Fig. 7: Adjusted autopilot dynamic which tangents the boundary of the RA zone

Nevertheless the time needed to capture the cleared flight level is increased as illustrated on the following figure which present the radar captured and simulated vertical profiles. It can be seen that the simulated vertical profile is 100 feet under the cleared flight level about 8 seconds later than the radar captured vertical profile. Dots represent the instants where an RA is issued onboard the radar captured trajectory, whereas the thin continuous line represents the altitude capture of the tuned autopilot: it can be seen that no RA appears.

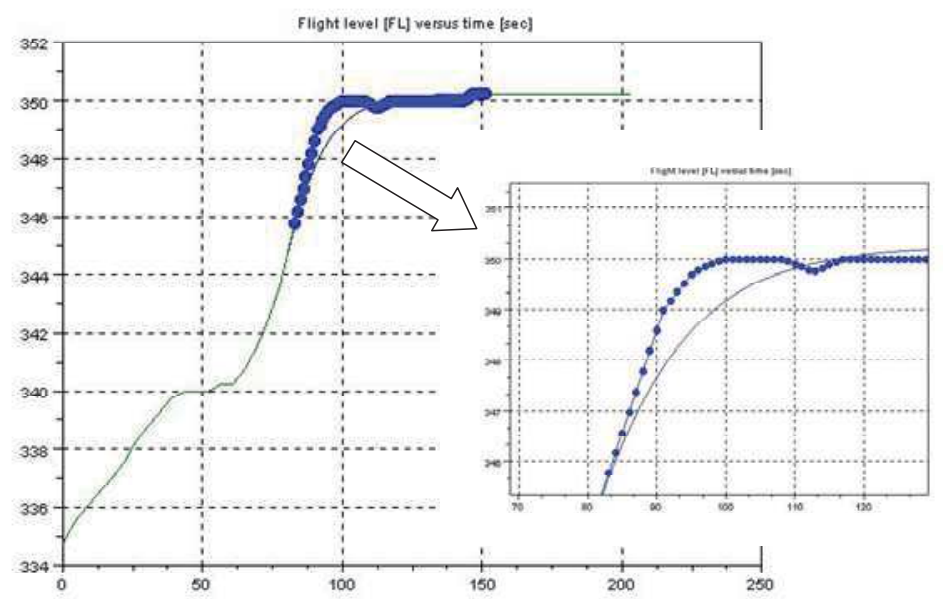

Fig. 8: Radar captured and simulated vertical profile

Using the actual TCAS version 7.0 logic on the simulated vertical profile shows that a Traffic advisory (TA) is still issued. But as expected no RA is issued.

\section{Conclusion}

In this paper, the TCAS resolution advisories generation process during level-off situation has been overviewed and illustrated on a real situation captured through mode S radar with RA downlink service. It has been emphasized that one common type of resolution advisories is that which is issued when aircraft are expected to level-off 1000 feet apart, and are converging at the same time. These resolution advisories, often subsequently classed as 'operationally unnecessary' from an air traffic control standpoint, can be perceived as disturbing by controllers and 
by pilots. This issue has been tackled by proposing a method to prevent RA triggering during level off situation. This method is based on the setting of the altitude at which the autopilot switches towards the altitude capture mode and on an intrinsic parameter of the autopilot altitude capture mode, namely its natural frequency.

Applying the proposed method to the illustrative example prevents the issuance of resolution advisories when aircraft are expected to level-off 1000 feet apart. The method which has been developed is one of the simplest options, and allows altitude capture without triggering resolution advisories for vertical speeds up to $3000 \mathrm{feet} / \mathrm{min}$. The time needed by the aircraft to capture the cleared flight level is increased.

Future work will focus on improving the time needed to capture the cleared flight level under the constraint to keep positive the distance between the autopilot altitude capture dynamic and the boundary of the RA zone. Also the investigation of the tuning of the altitude capture dynamic in order to deal with higher rates of climb/descent should be addressed. The altitude quantization and alpha - beta altitude tracker ${ }^{5}$ used by TCAS should be taken into account.

In addition, extending the proposed method to the cases where the intruder aircraft is not level has to be conducted. Does anybody wish to conduct real time experiments?

\section{Acknowledgments}

The authors wish to thank Jean-Marc Loscos and Christian Aveneau for helpful inputs and comments.

\section{References}

1 Aveneau C., TCAS : Traffic alert and Collision Avoidance System, over 15 years of work by DSNA to improve ATM safety, Revue Technique de la DTI no. 6 pp. 31-53 (2007) (http://www.dsna-dti.aviation-civile.gouv.fr/actualites/revuesgb/revue06gb/site_en/introduction.html)

2 Eurocontrol ACAS Programme, RAs and 1000ft level-off manoeuvres, ACAS II bulletin N², March 2003

3 Eshelby, M.E., Aircraft performance: Theory and Practice, AIAA Education Series, 2000

4 Khalil H.K., Nonlinear Systems, Third Edition, Prentice Hall, 2002

5 International Civil Aviation Organization, Annex 10, Volume IV, Surveillance Radar and Collision Avoidance Systems, Third Edition, July 2002

6 Ogata K., Modern Control Engineering, 4th Edition, Prentice Hall, 2001 